\title{
Holistic review of people with diabetes and chronic kidney disease reveals important multimorbidity and unmet clinical need: The ENHIDE diabetes renal telehealth pilot study
}

\author{
Authors: Peter H Winocour, ${ }^{A}$ Karen Moore-Haines, ${ }^{B}$ Keith Sullivan, ${ }^{C}$ Anne Currie, ${ }^{D}$ Andrew Solomon ${ }^{A}$ and \\ Dawn Hardy ${ }^{\mathrm{D}}$
}

\begin{abstract}
Diabetes and kidney disease commonly coexist and management is complex given frequent additional comorbidity. The East and North Herts Institute of Diabetes and Endocrinology (ENHIDE) renal diabetes telehealth project examined the feasibility of data extraction from primary care records for virtual consultant review as a prelude to a telehealth case-based discussion with primary care teams. Data extraction identified 2,356 cases from 16 general practices, of which 14 took part in a skype telehealth casebased discussion session. The service was well received by primary care as a workable means of delivering patient care. In addition, significant unmet clinical needs were identified with opportunities to empower patient selfmanagement of acute metabolic and foot issues, and better coordination of care between specialist diabetes and renal teams. The increasing clinical burden in all care settings and the commitment in the NHS plan for wider use of digital healthcare and streamlining of outpatient care highlight the need for service reconfiguration.
\end{abstract}

KEYWORDS: Diabetes mellitus, chronic kidney disease, telehealth, integrated care

DOI: 10.7861/clinmed.2019-0418

\section{Introduction}

Diabetes mellitus (DM) and chronic kidney disease (CKD) are closely linked, with CKD in $40 \%$ of individuals on diabetes registers, and diabetes considered the most common cause of end-stage renal failure. ${ }^{1,2}$

Authors: A consultant diabetologist, QEII Hospital, Welwyn Garden City, UK; ${ }^{\text {B }}$ project manager, QEII Hospital, Welwyn Garden City, UK; ${ }^{C}$ professor of statistics, institution, University of Hertfordshire, Hatfield, UK; ' Diabetes specialist nurse, QEII Hospital, Welwyn Garden City, UK
Given that ageing and obesity are implicated in DM and CKD and both are becoming more common, the magnitude of this comorbid disease cannot be underestimated. ${ }^{3,4}$

In addition to managing glycaemia and renal disease, assessment for cardiovascular disease and related modifiable risk factors, anaemia, foot, eye and bone health is required, with patient empowerment to ensure rapid access for acute metabolicrenal and active foot events. ${ }^{5}$

Primary care is the setting for the majority of those with DM and CKD, with perhaps no more than $20 \%$ under specialist diabetes and/or renal care. 6 Surveys of basic care processes and achievement of modifiable cardio-metabolic targets show deficiencies in quality care measures. ${ }^{1,7-9}$ Previous successful projects to carry out surveillance of renal disease and comorbid diabetes did not address all aspects of care. ${ }^{10,11}$ Integrated diabetes care with annual consultant diabetologist visits to primary care in operation in east and north Hertfordshire since 2010 identified large numbers of high risk cases requiring additional input.

The East and North Herts Institute of Diabetes and Endocrinology (ENHIDE) diabetes renal telehealth pilot project was initiated in December 2016 with objectives:

$>$ to examine the feasibility of extraction of more comprehensive datasets and trends from primary care diabetes registers than provided by the National Diabetes Audit

> to examine the feasibility of data utilisation for individualised patient care

$>$ to evaluate the practicality and acceptability to primary care of telehealth virtual reviews for both staff education and case management

$>$ to evaluate the extent of unmet clinical need

$>$ to create new sources of information to improve selfmanagement.

The study design has been outlined in detail elsewhere. ${ }^{12}$ All patients on diabetes registers were reviewed to identify those with pre-set criteria for $C K D$ - estimated glomerular filtration rate (eGFR) $<60 \mathrm{~mL} / \mathrm{min} / 1.73 \mathrm{~m}^{2}$ and/or urine albumin:creatinine ratio $(A C R)>10 \mathrm{mg} / \mathrm{mmol}$. ACR has marked variability, especially at categorical cut off thresholds. By choosing an ACR of $>10$ we 
minimised inclusion of those with marginal and variable ACR elevations. The east and north Hertfordshire area is a relatively affluent part of England with pockets of deprivation. The population is supported by 55 separate general practice services. We identified 20 practices representative of the wider population. In the current report 16 practices with most comprehensive data were reviewed and 2,147 patient details were analysed by two consultant diabetologists in three 4-hour weekly sessions.

The most contemporary measures of body mass index, blood pressure, lipid, haemoglobin A1c (HbA1c), eGFR, ACR, haemoglobin $(\mathrm{Hb})$, and assessment for anaemia were used and compared where possible with data for up to 2 years previous. Cardiovascular, foot and retinal status, and record of smoking and evaluation of metabolic bone health, where available, was recorded.

In addition, diabetes, lipid lowering, blood pressure and antiplatelet-anticoagulant medications, and any treatment for anaemia was recorded up to 3 months prior to the most contemporary measurements.

Standards of care (Box 1) and management guidance and referral criteria for specialist support based on local commissioned pathways and national guidelines (supplementary material S1) were used to evaluate clinically unmet need. ${ }^{13-19}$

\section{Results}

> Data were extracted from Systm1 and EMIS general practice data management systems. Significant time was required to secure this information and align with other patient identifiable measures from acute hospital and community specialist services, laboratory and retinal screening data sets. Read coded information on cardiovascular disease (CVD) and foot status was not stated in $74 \%$ and $5 \%$.

$>$ Sixteen practices participated and submitted data as requested. Of those practices that took part, 10,243 diabetes patients were identified, of which 2,356 (23\%) had CKD.

$>$ eGFR was $<60 \mathrm{~mL} / \mathrm{min} / 1.73 \mathrm{~m}^{2}$ in $1,888(80 \%)$ and $\mathrm{ACR}>10$ $\mathrm{mg} / \mathrm{mmol}$ in 820 (35\%). eGFR measures were not available in $42(1.85 \%)$, all of whom had raised ACR $>10 \mathrm{mg} / \mathrm{mmol}$ and ACR measurements were not available in 919 (39\%), all of whom had eGFR $<60 \mathrm{~mL} / \mathrm{min} / 1.73 \mathrm{~m}^{2}$. Of those 1,602 with both eGFR and ACR data 510 (32\%) had both eGFR $<60 \mathrm{~mL} / \mathrm{min} / 1.73 \mathrm{~m}^{2}$ and ACR $>10 \mathrm{mg} / \mathrm{mmol}$.

> Eighty-one per cent were solely managed in primary care, $14 \%$ attended the hospital or community specialist diabetes services ( $2 \%$ of whom also attended specialist renal services) and $5 \%$ only attended the specialist renal services in East and North Hertfordshire NHS Trust.

$>$ The mean age was 75 , with a roughly even gender split ( $48 \%$ female). Smoking status was recorded in 2,128 people, and of these $10 \%$ were current smokers and $43 \%$ were ex-smokers.

We carried out comprehensive evaluation in every case regardless of category in 3 practices, but for the remaining 13 practices we split the cohort into categories based on age and renal function and did not individually evaluate those in category 4 (Table 1).

\section{Box 1. Standards of care for diabetes with chronic kidney disease}

CKD: Identify persistent progressive decline in eGFR $>10 \mathrm{~mL} / \mathrm{min} / 1.73 \mathrm{~m}^{2}$ over 2 years.

ACR: Identify progressive increase from $>2.5 \mathrm{mg} / \mathrm{mmol}$ to $>10 \mathrm{mg} / \mathrm{mmol}$ (women) and $>3.5 \mathrm{mg} / \mathrm{mmol}$ to $>30 \mathrm{mg} / \mathrm{mmol}$ (men).

Blood pressure: $140 / 90 \mathrm{mmHg}(130 / 80 \mathrm{mmHg}$ if albuminuria present) attainment.

Non-HDL cholesterol: $2.5 \mathrm{mmol} / \mathrm{L}$ attainment.

HbA1c: 58-68 mmol/mol (7.5-8.5\%) attainment, especially in hypoglycaemia risk reduction.

Hypoglycaemia enquiry record.

Haemoglobin: Check if $<110 \mathrm{~g} / \mathrm{L}$ haematinic check and management if iron deficient; consider in HbA1c assessment.

Record Read-coded cerebrovascular disease status accurately.

Record Read-coded coronary artery disease and heart failure status accurately.

Foot care: Podiatry should be considered unless recorded as no neuropathy/peripheral vascular disease.

Aspirin (or alternative antiplatelet use) unless contraindicated, for those with albuminuria and/or CVD.

Record if BMI $>30 \mathrm{~kg} / \mathrm{m}^{2}$ and weight-reducing input and selection of weight-neutral or reducing therapies in diabetes and CKD with obesity.

Record of smoking cessation input for smokers.

Retinopathy status recorded in care plan and recognition of proliferative retinopathy/maculopathy with respect to glycaemic management.

Annual measurement of calcium, vitamin D and parathyroid hormone levels if CKD 3b or worse and use of activated vitamin D in normocalcaemic secondary hyperparathyroidism where parathyroid hormone 2-9 times the upper limit of the reference range.

Referral to renal department with deteriorating eGFR, eGFR $<30 \mathrm{~mL} / \mathrm{min} / 1.73 \mathrm{~m}^{2}$, nephrotic range proteinuria, unexplained anaemia, hyperparathyroidism, uncontrolled hypertension.

Referral to specialist diabetes service with active foot and retinal complications, recurrent hypoglycaemia, $\mathrm{HbA} 1 \mathrm{c}>75 \mathrm{mmol} / \mathrm{mol}$, especially if ACR $>30 \mathrm{mg} / \mathrm{mmol}$ and/or CKD $3 b\left(\right.$ eGFR $30-45 \mathrm{~mL} / \mathrm{min} / 1.73 \mathrm{~m}^{2}$ ).

$\mathrm{BMI}=$ body mass index; $\mathrm{CKD}=$ chronic kidney disease; $\mathrm{CVD}=$ cardiovascular disease; eGFR = estimated glomerular filtration rate; HbA1c = haemoglobin A1 c; $\mathrm{HDL}$ $=$ high-density lipoprotein; $\mathrm{ACR}=$ urine albumin:creatinine ratio. 
Table 1. Categorisation of chronic kidney disease

by age, estimated glomerular filtration rate and albumin:creatinine ratio

Category 1: Patients aged less than 75 years with an eGFR $<60 \mathrm{~mL} / \mathrm{min} / 1.73 \mathrm{~m}^{2}$

Category 2: Patients aged older than 75 years with an $23 \%$ eGFR $<45 \mathrm{~mL} / \mathrm{min} / 1.73 \mathrm{~m}^{2}$

Category 3: Patients aged older than 75 years with eGFR $>45 \mathrm{~mL} / \mathrm{min} / 1.73 \mathrm{~m}^{2}$ and $A C R>10 \mathrm{mg} / \mathrm{mmol}$

Category 4: Patients older than 75 years eGFR $>45$ $\mathrm{mL} / \mathrm{min} / 1.73 \mathrm{~m}^{2}$ and $\mathrm{ACR}<10 \mathrm{mg} / \mathrm{mmol}$

$\mathrm{ACR}=$ albumin creatinine ratio; $\mathrm{eGFR}=$ estimated glomerular filtration rate.

Practices were encouraged to provide standard recommendations on sick day rules, bone health checks, smoking cessation and podiatry for those in category 4 (Table 1). Hand-held sick-day guidance cards were made available for all identified patients.

An in-depth virtual review of all available biomedical patterns and medications was carried out in 2,147 of the 2,356 people, referral or other actions identified in individualised care plans were sent to the practice. The number of recommendations for changes in management or specialist referral is shown in Fig 1.

\section{Telehealth virtual case reviews}

Following consultant comprehensive review of all suitable cases, a 2-hour Skype telehealth educational session was agreed by 14 of the 16 participating practices. Prior identification of 20 cases with a spectrum of clinical scenarios was sent to the practice enabling case-based discussions.

\section{Renal function}

The pattern of eGFR over at least 2 years was possible with comparative date in 1,677 people. Based on the described criteria, clinically significant deterioration was noted in 380 (23\%), was stable in 592 (35\%), variable in 552 (33\%) and potentially improved in 153 (9\%).

The pattern in ACR over at least 2 years was possible with comparative date in 1,437 people. Deterioration in category was noted in $344(24 \%)$, improved in $72(5 \%)$, stable in $626(44 \%)$, and variable in $395(27 \%)$. In this group $387(27 \%)$ had an ACR >30 $\mathrm{mg} / \mathrm{mmol}$ and $27(2 \%)$ had an ACR $>300 \mathrm{mg} / \mathrm{mmol}$. Based on these findings 351 (16\%) were identified who required referral to renal department for further evaluation.

\section{Blood pressure}

We obtained blood pressure readings from 2,142 people, of which 1,258 (59\%) were already taking blood pressure treatment. Based on the criteria of target blood pressure and selection of appropriate agents based on albuminuria status, changes in blood pressure medication were recommended in $27 \%$.

Lipids

Total cholesterol measurements were available in 2,249 cases and missing in 107 cases. Of those with data $80 \%$ had the last serum cholesterol recorded as $<5 \mathrm{mmol} / \mathrm{L}$. High-density lipoprotein (HDL) cholesterol measures were available in 1,546 cases with no record in 810 . Of those with data, only $32 \%$ had non-HDL cholesterol value at the target $2.5 \mathrm{mmol} / \mathrm{L}$ or less. Based on the non-HDL cholesterol target, changes in lipid lowering medication were recommended in 39\% (923 cases).

\section{Glycaemia}

$\mathrm{HbA1c}$ baseline values were available in 2,356 and 2-year trends in 2,264 people. Patterns of HbA1c and hypoglycaemia risk have been analysed in detail elsewhere. ${ }^{6}$ Five-hundred and seventy-six were on insulin with another 114 also on sulphonylureas in addition to insulin, 458 on sulphonylureas and other non-insulin therapies, 508 were solely on diet and 700 were on alternative agents alone or in combination without insulin and/or sulphonylureas. Median

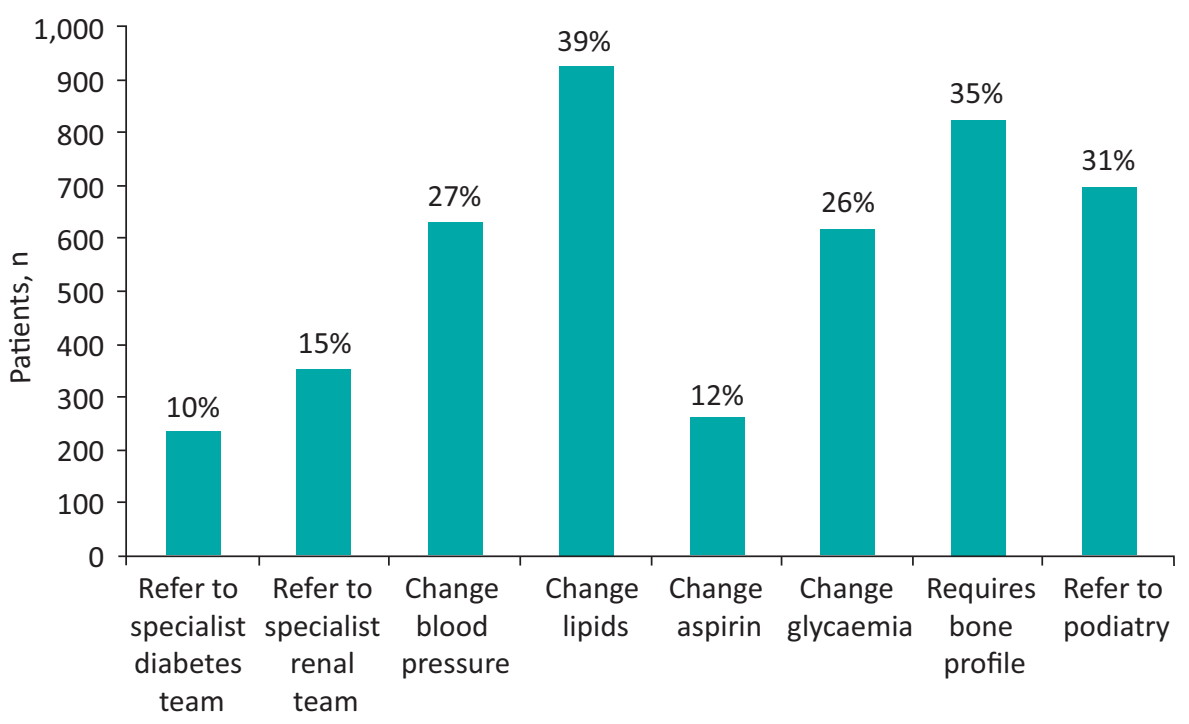

Recommendation
Fig 1. Number of cases where recommendations for changes in care or specialist referral were made. 
$\mathrm{HbA1c}$ was $53 \mathrm{mmol} / \mathrm{mol}$ and 1,493 (63\%) had HbA1c levels at $58 \mathrm{mmol} / \mathrm{mol}$ or less. We identified $236(10 \%)$ where referral to specialist diabetes care was suggested on the basis of glycaemia control and moderate renal disease (eGFR 30-45 mL/min/1.73 m² and or ACR $>30 \mathrm{mg} / \mathrm{mmol}$ ). Changes in glycaemic medication dosage or category were suggested in 651 people (28\%).

\section{Haemoglobin}

Contemporary $\mathrm{Hb}$ measures were available in 2,273 people with a mean value of $128 \mathrm{~g} / \mathrm{L}$. Of these $385(17 \%)$ were anaemic with $\mathrm{Hb}$ value at $110 \mathrm{~g} / \mathrm{L}$ or less. There was an additional 345 (15\%) coded as anaemic on records who were on therapy for anaemia with $\mathrm{Hb}$ values $>110 \mathrm{~g} / \mathrm{L}$.

\section{Body mass index}

Information on body mass index was calculated in 2,237 people, of whom 1,082 (48\%) were categorised as obese with levels $>30 \mathrm{~kg} / \mathrm{m}^{2}$.

\section{Cardiovascular disease}

Read coding was not recorded in $74 \%$, despite documentation of CVD elsewhere in some records, so there was less clarity as to whether non recording meant lack of CVD. Where coding was available, there was a record of ischaemic heart disease in primary care medical records in 603 (26\%) and of stroke-cerebrovascular disease in 301 (13\%).

\section{Feet and retinal status}

Of the 2,356 people, foot examination was declined or not possible through amputation in 117-126 feet. Of those remaining cases, $56 \%$ were categorised as low risk, $43 \%$ were moderate to high risk and $1 \%$ had active foot disease-ulceration. Foot advice through patient hand-held information cards and/or referral to podiatry was recommended for 721 people (31\%) with high risk for active foot problems.

Retinal screening information was available in 2,182 people of whom 924 (42\%) had any degree of retinopathy, with background retinopathy in the majority, 648 (30\% of the total screened).

\section{Use of antiplatelet/anticoagulant therapy}

This was advocated for established CVD (including atrial fibrillation) and those with increased CVD risk based on highlevel albuminuria, based on the consensus recommendations of the Joint British Societies for prevention of cardiovascular disease (JBS3). ${ }^{16}$ Overall 1,288 (55\%) were on either antiplatelet or anticoagulant therapy. Addition of such therapy was recommended in a further $272(12 \%)$.

\section{Bone health profile}

Serum calcium measures had been carried out in 725 (31\%), vitamin D in 225 (10\%) and parathyroid hormone in 204 (9\%) of the total group of 2,356 people. National recommendations state parathormone measurement when eGFR is $45 \mathrm{~mL} / \mathrm{min} / 1.73 \mathrm{~m}^{2}$ or lower, and we identified 830 (35\%) where this had not been carried out.

\section{Discussion}

The combination of DM and CKD currently affects over $2 \%$ of the adult population and is increasing in incidence. ${ }^{1,13}$ An abundance of data shows substandard care, regardless of location. Previous surveillance models focused specifically on renal function. ${ }^{10,11}$ The complex multimorbid nature of DM CKD requires a fundamental change to the model of care.

The 10-year NHS plan highlights the need to reduce traditional outpatient services and use digital technology to improve care. ${ }^{21}$ Interventions need to be structured, intensive and multifaceted to address DM CKD. Services need expertise from different medical disciplines working closely with primary care, improved selfmanagement and primary care upskilling. This current programme established a basis to deliver these requirements.

One objective was to examine the feasibility of extraction of comprehensive data sets from primary care. This was fraught with access difficulties and identified the need for streamlined real time data system monitoring, and clerical support to update and code core data on individual care records. This was most clearly recognised with incomplete coding for CVD and frequent omission of ACR measurements.

Consultant diabetologists provided individualised patient care in this pilot through manual case review, but this was time consuming. Streamlined care planning through a clinical algorithm for primary care could identify unstable or high-risk scenarios. The use of Skype consultations was well received in the format described, and upskilled primary care through virtual case-based discussion.

The project demonstrated that $16 \%$ of patients with DM and CKD attend hospital DM and/or renal services. Other virtual modelling renal services showed many people with CKD can receive surveillance without routine clinic appointments. ${ }^{10,11}$ We also found that $1.6 \%$ were under both renal and diabetes clinics with an opportunity to avoid duplication of unnecessary appointments.

The creation and distribution of patient-held cards for sickday rules, metabolic care and foot risk advice were designed to empower patients to seek prompt clinical input when necessary. In addition, the pilot demonstrated important unmet clinical need.

The previous National Diabetes Audit and other international surveys of attainment of core biomedical targets have shown that glycaemia, blood pressure and lipid rates are not being attained. ${ }^{1,7-9}$ This partly reflects organisation of care, but may also reflect issues with medication compliance and efficacy, and recognition that targets may not be attainable in many cases. ${ }^{22}$

We identified important diabetes and renal issues that had not been previously addressed but which met the criteria for specialised input based on commissioned care pathways. The majority of those with DM CKD will not progress to end-stage renal disease but will be at major CVD retinal and foot risk, and require nuanced glycaemic management. ${ }^{17,23}$ Optimisation of blood pressure and lipid lowering medication was frequently recommended. A less recognised consideration was for initiation of antiplatelet therapy in those with evident CVD-proteinuria CKD where increased thrombotic risk had not been addressed.

The ENHIDE study also confirms that DM CKD requires a wider holistic approach. ${ }^{5}$ The prevalence of obesity in almost $50 \%$ highlighted the opportunity to utilise new classes of treatment for diabetes for those with suboptimal glycaemic control that achieve weight reduction and improve cardiorenal outcome. ${ }^{17,24,25}$ 
Forty-one per cent of DM CKD had at-risk feet. Highlighting this and enabling patient self-care provided a vital opportunity for early presentation, given the time critical nature of acute foot issues.

Retinopathy was not ubiquitous among those with CKD. The absence of retinopathy might alert the possibility of modifiable non-DM renal disease, especially with albuminuria. The absence of retinopathy and albuminuria might indicate microvascular damage is not the basis for CKD, and there is evidence that renal function does not progress so rapidly in that situation.

Unmet clinical need for metabolic bone health is less surprising. Recommendations for assessment and treatment have been targeted at renal specialist teams and not cascaded to primary care. ${ }^{14}$ Anaemia is another factor neglected in assessment of DM CKD. It is common with over $30 \%$ in the current cohort. It has adverse prognostic import, and requires assessment. ${ }^{15}$ Assessment of glycaemic control through measurement of $\mathrm{HbA} 1 \mathrm{c}$ may be misleading. ${ }^{6}$ Exclusion of blood loss and assessment for erythropoietin or iron deficiency need consideration. Anaemia will predominate among the older population with CKD DM and is a factor in increased morbidity and mortality with heart failure. New therapeutic options for managing heart failure progression with gliflozins could be considered, or conservative care can be planned in the frailest elderly. ${ }^{24}$

In summary this pilot project demonstrates it is possible to identify patients with DM CKD in primary care in large numbers, to individualise care, to identify unmet clinical needs requiring escalation or de-escalation of therapy in primary care, with specialist review in up to $20 \%$ of people with DM and CKD. Ongoing evaluation is necessary to assess the extent and durability of clinical benefit.

We have utilised a new approach to care which, if extended from this pilot, offers the potential for effective multimorbid integrated care between primary care and different specialists, using digital technology to reduce unnecessary clinic activity, a key deliverable of the NHS 10-year plan.

\section{Supplementary material}

Additional supplementary material may be found in the online version of this article at www.rcpjournals.org/content/clinmedicine: S1 - Management guidance for comprehensive care of diabetes and chronic kidney disease.

\section{Conflicts of interest}

Dr Peter $\mathrm{H}$ Winocour has received honoraria for delivering educational meetings and/or attending advisory boards for Astra Zeneca, Boehringer Ingelheim, Eli Lilly, MSD, Napp, Sanofi, Novo and Vifor Pharmaceuticals.

\section{Funding}

This project was funded by the NHS East and North Hertfordshire Clinical Commissioning Group with additional support from an unrestricted medical education grant from Sanofi Aventis towards diabetes specialist nurse salary costs.

\section{References}

1 Hill C], Cardwell CR, Patterson CC et al. Chronic kidney disease and diabetes in the National Health Service: a cross-sectional survey of the UK National Diabetes Audit. Diabetic Med 2014;31:448-54.
2 Steenkamp R, Caskey F. UK Renal Registry 18th annual report: Chapter 6. Comorbidities and current smoking status amongst patients starting renal replacement therapy in England, Wales and Northern Ireland from 2013 to 2014. Nephron 2016;132(suppl 1):145-54.

3 Ellam T, Twohig H, Khwaja A. Chronic kidney disease in elderly people: disease or disease label. BMJ 2016;352:h6559.

4 Hill C], Cardwell CR, Maxwell AP et al. Obesity and kidney disease in type 1 and type 2 diabetes: an analysis of the National Diabetes Audit. QJM 2013;106:933-42.

5 Winocour PH. Diabetes and chronic kidney disease: an increasingly common multi-morbid disease in need of a paradigm shift in care. Diabetic Med 2018;35:300-5.

6 Winocour PH, Moore-Haines K, Sullivan K et al. HbA1c in a primary care cohort with diabetes and chronic kidney disease: the ENHIDE diabetes renal telehealth project. $\mathrm{Br}$ ] Diabetes 2019:[submitted].

7 Luk AO, Li X, Zhang Y et al. Quality of care in patients with diabetic kidney disease in Asia: The Joint Asia Diabetes Evaluation (JADE) Registry. Diabetic Med 2016;33:1230-9.

8 De Cosmo S, Viazzi F, Pacilli A et al. Achievement of therapeutic targets in patients with diabetes and chronic kidney disease: insights from the Associazione Medici Diabetologi Annals initiative. Nephrol Dial Transplant 2015;30:1526-33.

9 Nitsch D, Caplin B, Hull SA et al. National chronic kidney disease audit: National report (Part 1). Healthcare Quality Improvement Partnership, 2017.

10 Harnett P, Jones M, Almond M, Ballasubramanian G, Kunnath V. A virtual clinic to improve long term outcomes in chronic kidney disease. Clin Med 2018;18:356-63.

11 Rayner HC, Hollingworth L, Higgins R, Dodds S. Systematic kidney disease management in a population with diabetes mellitus: turning the tide of kidney failure. BMJ Qual Saf 2011;20:903-10.

12 Winocour PH, Moore-Haines K, Solomon A, Currie A, Hardy D. ENHIDE telehealth primary care support of adults with diabetes and chronic kidney disease. A pilot study - rationale and study design. $\mathrm{Br}$ J Diabetes 2019;19:53-62.

13 National Institute for Health and Care Excellence. Chronic kidney disease in adults: assessment and management: Clinical guideline [CG182]. NICE, 2015

14 Ketteler M, Block GA, Evenepoel P et al. Diagnosis, evaluation, prevention, and treatment of chronic kidney disease-mineral and bone disorder: synopsis of the kidney disease: Improving Global Outcomes 2017 clinical practice guideline update. Ann Intern Med 2018;168:422-30.

15 Padhi S, Glen J, Pordes ABAJ, Thomas ME, Guideline Development Group. Management of Anaemia in chronic kidney disease: summary of updated NICE guidance. BMJ 2015;350:32-6.

16 JBS3 Board. Joint British Societies' consensus recommendations for the prevention of cardiovascular disease (JBS3). Heart 2014;100(Suppl 2):ii46-51.

17 Winocour PH, Bain SC, Chowdhury T et al. Managing hyperglycaemia in patients with diabetes and diabetic nephropathy - chronic kidney disease. Summary of recommendations 2018. Br J Diabetes 2018;18:78-89.

18 Mark PB, Winocour P, Day C. Management of lipids in adults with diabetes mellitus and nephropathy and/or chronic kidney disease: summary of joint guidance from the Association of British Clinical Diabetologists (ABCD) and the Renal Association (RA). $\mathrm{Br}]$ Diabetes 2017:17:64-72.

19 Dasgupta I, Banerjee D, Chowdhury TA et al. Association of British Clinical Diabetologists (ABCD) and Renal Association clinical guidelines: Hypertension management and reninangiotensin-aldosterone system blockade in patients with diabetes, nephropathy and/or chronic kidney disease. Summary of recommendations. Br ] Diabetes 2017;17:160-4.

20 Holman N, Forouhi NG, Goyder E, Wild SH. The Association of Public Health Observatories (APHO) Diabetes Prevalence Model: 
estimates of total diabetes prevalence for England, 2010-2030. Diabet Med 2011;28:575-82.

21 NHS England. The NHS Long Term Plan. NHS, 2019.

22 Winocour PH. Effective diabetes care - a need for realistic targets. BMJ 2002;324:1577-80.

23 Finne P, Groop PH, Arffman M et al. Cumulative risk of end-stage renal disease among patients with type 2 diabetes: A nationwide inception cohort study. Diabetes Care 2019;42:539-44.

24 Zelniker TA, Wiviott SD, Raz I et al. SGLT2 inhibitors for primary and secondary prevention of cardiovascular and renal outcomes in type 2 diabetes: a systematic review and meta-analysis of cardiovascular outcome trials. Lancet 2019;393:31-9.
25 Gerstein HC, Colhoun HM, Dagenais GR et al. Dulaglutide and renal outcomes in type 2 diabetes: an exploratory analysis of the REWIND randomised placebo-controlled trial. Lancet 2019;394:131-8.

Address for correspondence: Dr Peter H Winocour, ENHIDE, Howlands Clinic, QEII Hospital, Welwyn Garden City, Hertfordshire AL7 4HQ, UK.

Email: peter.winocour@nhs.net

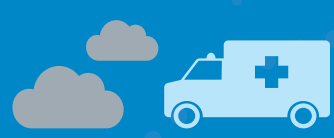

\section{Outpatients: the future - adding value through sustainability}

Published in 2018, this report from the Royal College of Physicians argues for new models of outpatient care.

\section{Download the report and} supplementary case studies at www.rcplondon.ac.uk/outpatients-future-sustainability 